九州大学学術情報リポジトリ

Kyushu University Institutional Repository

\title{
REPRESENTATION THEOREMS AND PRIMITIVE PREDICATES FOR LOGIC PROGRAMS
}

Yokomori, Takashi

International Institute for Advanced Study of Social Information Science, Fujitsu Limited

https://doi.org/10.5109/13374

出版情報: Bulletin of informatics and cybernetics. 22 (1/2), pp.19-37, 1986-03. Research Association of Statistical Sciences

バージョン :

権利関係 : 


\title{
REPRESENTATION THEOREMS AND PRIMITIVE PREDICATES FOR LOGIC PROGRAMS
}

\author{
By
}

\author{
Takashi YokomorI*
}

\begin{abstract}
We present several representation theorems for logic programs in terms of formal grammatical formulation. First, for a given logic program $P$ the notion of the success language of $P$ is introduced, and based on this language theoretic characterization of a logic program several types of representation theorems for logic programs are provided. Main results include that there effectively exists a fixed logic program with the property that for any logic program one can find an equivalent logic program such that it can be expressed as a conjunctive formula of a simple program and the fixed program. Further, by introducing the concept of an extended reverse predicate, it is shown that for any logic program there effectivily exists an equivalent logic program which can be expressed as a conjunctive formula consisting of only extended reverse programs and append programs.
\end{abstract}

\section{Introduction}

Since, needless to say the original work of Colmerauer and Kowalski ([1] and [9]), a recent world-wide trend on FGCS conception has been one of the primary subjects, there are numerous work on logic programming languages and the theory of logic programs. It is well accepted that, among others, the research on a subset of firstorder predicate logic called Horn clause logic has taken the central position in this area because of its importance of providing an interesting formal computation model for a programming language PROLOG. As is well-known, PROLOG, based on the procedural interpretation to Horn clause logic, has an operational semantics determined by the resolution principle. In the context of the semantics of predicate logic as a programming language van Emden and Kowalski ([3]) have studied on model-theoretic, operational and fixedpoint semantics of logic programs, while using a Turing machine formulation Shapiro ([11]) has defined and argued a kind of model-theoretic semantics of logic programs.

In this paper we are concerned with establishing several representation theorems for "logic programs (Horn clause programs)" in terms of formal language theoretic formulation. In course of the formal grammatical treatment of logic programs we introduce the notion of the success language of a logic program over a finite alphabet,

* International Institute for Advanced Study of Social Information Science, Fujitsu Limited 150 Miyamoto, Numazu, Shizuoka 410-03 JAPAN 
which turns out to be another way of providing a model-theoretic semantics for logic programs. Here, by formal grammars we mean generative grammars of Chomsky, and it should be remarked that the theory of formal languages (e.g. [6], [8] and [10]) has been well-developed enough in itself to make a lot of contributions to other research areas such as the theory of logic programming. This view may be supported, for example, when we think of the similarity between the refutation process in logic programs and the derivation steps in context-free grammars, and note that logic programs can be regarded as a kind of an extention of context-free grammars. In fact, Shapiro investigates the computational complexity of logic programs using the similarity of their operational behaviors to those of alternating Turing machines. ([11])

With the help of an encoding technique it is shown that one can associate a logic program with a formal language (the success language mentioned above) over a finite alphabet. This leads to a semantic characterization of logic programs as previously mentioned, although that is not our primary concern in the current paper. This kind of semantic approach to logic programs has been already preceeded by the paper [13]. It has been shown that any recursively enumerable language can be specified as a conjunctive formula of two deterministic logic programs and one simple logic program that serves as a mapping on the set of words. The work in this paper is motivated by the result above and extends it to present a variety of the ways of representing logic programs.

In this paper we present several representation theorems for logic progams which assert that there effectively exists a fixed logic program (we may call it generator program) with the property that for any logic program one can find an equivalent logic program such that it can be expressed as a conjunctive formula of a simple program and the fixed program.

Further, by analysing components in the representation results, it is shown that the "filtering function" serving as a homomorphism mapping and the "merging function" are sufficiently primitive in the sense that for any logic program there is an equivalent logic program which can be expressed within the use of combination of these two programs. By introducing the concept of "extended reverse", it is also proved that for any logic program one can find an equivalent logic program expressed as a conjunctive formula consisting of only "extended reverse" programs and "append" programs.

This paper is organized as follows. Section 2 is concerned with terminology, basic notions and results needed through the paper. In Section 3 several representation theorems for logic programs are established. Section 4 deals with the problem of what operations (predicate) is primitive for the representation formula obtained in Section 3. Concluding remarks and the future reseach direction are briefiy given in Section 5 . Appendix provides a proof for Lemma 3.4 which is used in the text to derive a representation result of logic programs. 


\section{Preliminaries}

\subsection{Formal grammars and their languages}

We now introduce a generative device which plays the main role in all of subsequent sections in this paper.

Definition. A generative grammar is an ordered quadruple $G=\left(N, T, P, S_{0}\right)$ where $N$ and $T$ are disjoint finite alphabets, $S_{0}$ is in $N$, and $P$ is a finite set of production rules of the form $Q_{1} \rightarrow Q_{2}$ such that $Q_{2}$ is a word over the alphabet $V=N \cup T$ and $Q_{1}$ is a word over $V$ containing at least one symbol of $N$. The elements of $N$ are called nonterminals and those of $T$ terminals; $S_{0}$ is called the initial symbol.

A word $u$ generates directly a word $v$, in symbols, $u \Rightarrow v$, if and only if there are words $u^{\prime}, u^{\prime \prime}, Q_{1}, Q_{2}$ such that $u=u^{\prime} Q_{1} u^{\prime \prime}, v=u^{\prime} Q_{2} u^{\prime \prime}$ and $Q_{1} \rightarrow Q_{2}$ belongs to $P$. Thus, $\Rightarrow$ is a binary relation on the set $V^{*}$ (the set of all words over $V$ including empty word e). We denotes $V^{*}-\{\mathrm{e}\}$ by $T^{+}$. Let $\Rightarrow^{*}$ be the reflexive, transitive closure of $\Rightarrow$. The language $L(G)$ generated by $G$ is defined by

$$
L(G)=\left\{w \text { in } T^{*} \mid S_{0} \Rightarrow * w\right\}
$$

$L(G)$ is called a language over $T$ (or on $T^{*}$ ).

Grammars are, in general, classified by the form of production rules, which yields a hierarchy of corresponding language families.

Definition. A generative grammar is also called phrase structure grammar. Let $G=\left(N, T, P, S_{0}\right)$ be a phrase structure grammar. Then, $G$ is called

(i) context-free if each production rule is of the form $X \rightarrow Q$, where $X$ in $N$, and $Q$ in $V^{*}$,

(ii) regular if each production rule is one of the two forms $X \rightarrow a$ or $X \rightarrow a Y$, where $a$ in $T$ and $X, Y$ in $N$, with the possible exception on the production rule $S_{0} \rightarrow e$ whose occurrence in $P$ implies that $S_{0}$ does not occur on the right hand side of any rule in $P$.

Definition. (1) Let $G=\left(N, T, P, S_{0}\right)$ be a context-free grammar with the property that (i) every rule in $P$ is of the form $A \rightarrow a x$, where $A$ in $N, a$ in $T, x$ in $N^{*}$, and (ii) for all $A$ in $N, a$ in $T, A \rightarrow a x$ and $A \rightarrow a y$ in $P$ implies $x=y$. Then, $G$ is called simple deterministic.

(2) A context-free grammar $G=\left(N, T, P, S_{0}\right)$ is called linear if $P$ consists of the rules of the form: $A \rightarrow u B v$, or $A \rightarrow w$, where $A, B$ in $N, u, v, w$ in $T^{*}$.

Definition. Let $L$ be a subset of $T^{*}$ for some alphabet $T$, and let $X$ be in \{phrase structure, context-free, simple deterministc, linear, regular\}. Then, $L$ is called an $X$ language if $L=L(G)$ for some $X$ grammar $G$. Further, a language generated by a phrase structure grammar is also called recursively enumerable.

Let $r \geq 1$ and $T_{r}=\left\{a_{1}, \cdots, a_{r}\right\}$. Further, Let $G r=\left(\left\{S_{0}\right\}, T, P, S_{0}\right)$ be a context-free grammar, where $T=T_{r} \cup\left\{\tilde{a} \mid a\right.$ in $\left.T_{r}\right\}$, and $P=\left\{S_{0} \rightarrow S_{0} a_{i} S_{0} \tilde{a}_{i} S_{0} \mid 1 \leq i \leq r\right\} \cup\left\{S_{0} \rightarrow e\right\}$. Then, $L\left(G_{r}\right)$ is called the Dyck language over $T_{r}$ and denoted by $D_{r}$.

Definition. Let $T$ be an alphabet. For each $a$ in $T$, let $f(a)$ be a word (possibly over a different alphabet from $T)$. Then, let $f(e)=e, f(x y)=f(x) f(y)\left(x, y\right.$ in $\left.T^{*}\right)$. The mapping $f$ is extended to the power set of $T^{*}$ as follows: for each $L$ over $T, f(L)=$ 
$\{f(w) \mid w$ in $L\}$. The mapping $f$ is called a homomorphism on $T^{*}$. Let $f$ be a homomorphism on $T^{*}$, and let $K$ be the alphabet of the range of $f$. Then, a mapping $f^{-1}$ defined by

$$
\text { for } L \text { over } K, f^{-1}(L)=\left\{w \text { in } T^{*} \mid f(w) \text { in } L\right\},
$$

is called the inverse homomorphism of $f$.

Definition. A homomorphism $f$ on $T^{*}$ is called

(1) a coding if for each $a$ in $T, f(a)$ is a symbol,

(2) a weak coding if for each $a$ in $T, f(a)$ is either a symbol or the empty word $e$,

(3) a weak identity if for each $a$ in $T, f(a)$ is either the symbol $a$ itself or the empty word $e$.

DEFINITION. A deterministic generalized sequential machine (dgsm) with accepting states is a 6-tuple $A=\left(Q, T, D, d, q_{0}, F\right)$, where

$Q$ : a finite set of states, $T:$ a finite set of input symbols, $D:$ a finite set of output symbols, $d$ : transition function from $Q \times T$ to $Q \times D^{*}, q_{0}$ : the initial state

in $Q$, and $F$ : a subset of $Q$ (a set of final states).

The function $d$ is extended to $Q \times T^{*}$ as follows: for $q$ in $Q, x$ in $T^{*}, a$ in $T$,

where

$$
\begin{aligned}
& d(q, e)=(q, e), \\
& d(q, a x)=(r, y)
\end{aligned}
$$

$$
\begin{gathered}
y=w_{1} w_{2} \\
d(q, a)=\left(p, w_{1}\right), d(p, x)=\left(r, w_{2}\right) \quad \text { for some } p \text { in } Q, w_{1}, w_{2} \text { in } D^{*} .
\end{gathered}
$$

Let $f_{A}$ be a mapping defined by

$$
f_{A}(x)=y \text { iff } d\left(q_{0}, x\right)=(p, y) \quad \text { for some } p \text { in } F .
$$

The mapping $f_{A}$ so defined is called a dgsm mapping of $A$.

Notation. Let $T$ be a finite alphabet. For a word $w=a_{1} \cdots a_{n}(n \geq 0)$ in $T^{*}$, the $(\sim)$-version $\tilde{w}$ denotes $\tilde{a}_{1} \cdots \tilde{a}_{n}$. Further, $w^{R}$ denotes the reverse $a_{n} \cdots a_{1}$.

\subsection{Logic programs and their languages}

This subsection introduces the concepts of a logic program and its associated language we shall deal with in the subsequent sections. We assume the reader to be familiar with the rudiments of mathematical logic.

Definition. A logic program is a finite set of Horn clauses, which are universally quantified logical sentences of the form

$$
A \leftarrow B_{1}, \cdots, B_{n} \quad(n \geq 0)
$$

where the $A$ and the $B$ 's are atomic formulae. In the above clause (C) $A$ is called the clause's head, while $B$ 's are called the clause's body. If $n=0$, then we simply donote it by $A$ instead of $A \leftarrow$.

Atomic formulae occurring in a logic program are called goals. A program is said to be dominated by a goal if the predicate name of the goal occurs only once as the head of a clause in the program.

Notational Convention (i) We use upper-case letters such as $X, Y, Z$ for variable 
symbols and lower-case letters such as $x, y, z$ for ground terms. For terms, letters $t, s, r$ are often used. The boldface versions like $\boldsymbol{P}, \boldsymbol{Q}$ are used for logic programs, while normal upper-case letters like $P, Q$ are used for goals, and lower-case letters $p, q$ for goal names.

(ii) For a logic program dominated by a goal, we sometimes refer to the program in terms of the name of the goal. In such a case it is assumed that the program name is the capital letter $\boldsymbol{P}$ of the goal name " $p$ ".

Definition.

Let $\boldsymbol{P}$ be a logic program and $Q$ a goal. If there is a refutation of a goal $Q$ from $\boldsymbol{P}$, then we say $\boldsymbol{P}$ succeeds on $Q$, or $Q$ succeeds (in $\boldsymbol{P}$ ).

In this paper we are concerned with logic programs whose data domains are finitely generated by a fixed set of symbols.

Definition. Let $\boldsymbol{P}$ be a logic program. The Herbrand universe of $\boldsymbol{P}$ is the set of all ground terms constructable from the set of constants $C$ and the set of function symbols $F$ occuring in $\boldsymbol{P}$, and we denote it by $D(F, C)$. Then, a logic program $\boldsymbol{P}$ is called a logic program over $C$ if $F$ comprises only one function symbol, and its Herbrand universe is denoted by $D(C)$.

As shown below, any Herbrand universe for a logic program can be coded in an appropriate manner into the domain $D(T)$ constructed from some fixed finite set of symbols $T$. In other words, any ground term which possibly appears in a program can be taxen as a word over some finite alphabet $T$.

LEMMA 2.1 There exist a fixed finite set of symbols $T$ and a one-to-one mapping $f$ such that for any logic program $\boldsymbol{P}$ with the domain $D(F, C)$ and for any goal $p\left(X_{1}, \cdots\right.$, $X_{n}$ ) there exist a logic program $\boldsymbol{P}^{\prime}$ with the domain $D(T)$ and a goal $P^{\prime}(X)$ with the property that $\boldsymbol{P}$ succeeds on $p\left(x_{1}, \cdots, x_{n}\right)$ iff $\boldsymbol{P}^{\prime}$ succeeds on $p^{\prime}(x)$, where $x=f\left(x_{1}, \cdots, x_{n}\right)$, PROOF. Let $g_{1}, g_{2}, \cdots$ be an enumeration of all function symols occurring in $D(F, C)$ of $\boldsymbol{P}$. (Note that a constant $k$ can be taken as a 0 -ary function symbol as in $k()$ ).)

Introduce a mapping $c$ from the set $D(F, C)$ to the set of lists as follows :

for a term $t=g_{i}\left(s_{1}, \cdots, s_{m}\right)(m \geq 0)$,

$$
c(t)=\left[\%, @^{i}, \$, s, c\left(s_{1}\right), \cdots, c\left(s_{m}\right), \hat{\$}\right] \text {. }
$$

wherh "[" and "]" are the list notation, $\$, \hat{\$}$, (a), $\%$ are new symbols, and (a) ${ }^{i}$ denotes a sequence (a), $\cdots$, (a) of $i$ (a)s. Further, for an $n$-tuple of terms $\left(t_{1}, \cdots, t_{n}\right)$, let $f$ be defined by

$$
f\left(t_{1}, \cdots, t_{n}\right)=\text { flatten }\left(\left[c\left(t_{1}\right), \#, \cdots, \neq, c\left(t_{n}\right)\right]\right),
$$

where "fiatten" is a mapping of fiattening lists,

\# is a new symbol (argument separator).

Define $p^{\prime}(X)$ as follows:

$$
p^{\prime}(X) \leftarrow f a t\left(X_{1}, \cdots, X_{n}, X\right), p\left(X_{1}, \cdots, X_{n}\right) \cdots\left(C_{0}\right)
$$

where $\operatorname{flat}\left(X_{1}, \cdots, X_{n}, X\right)$ succeeds iff $X=f\left(X_{1}, \cdots, X_{n}\right)$.

Further, let $\boldsymbol{P}^{\prime}$ be $\boldsymbol{P} \cup\left\{C_{0}\right\}$. Then, it is easily seen that $\boldsymbol{P}^{\prime}$ succeeds on $p^{\prime}\left(f\left(x_{1}, \cdots, x_{n}\right)\right)$ iff $\boldsymbol{P}$ succeeds on $p\left(x_{1}, \cdots, x_{n}\right)$ for $x_{i}$ in $D(F, C)$. Let $T=\{\sharp, \$, \hat{\$}$, (a), $\%, N I L\}$, where 
NIL denotes empty list, then $D(T)$ is the set of lists constructed from $T$ and the unique function symbol of the list constructor. Obviously, this satisfies the desired conditions.

Thus, it is sufficient for general discussion to deal with only logic programs over some fixed finite set.

Conventions. (1) In what follows, it may be assumed that (i) a logic program over $T$ has the domain of all lists constructed from a finite set of constants $T$, and (ii) otherwise specified, a goal is assumed to be a 1-ary predicate.

(2) As a notetion, given a finite set of symbols $T$ and a word $w=a_{1} \cdots a_{n}$ on $T^{*}$, the boldface $\boldsymbol{w}$ denotes the list version $\left[a_{1}, \cdots, a_{n}\right]$.

Logic programs together with goals are classified by the types of their associated languages.

Definition. Let $\boldsymbol{P}$ be a logic program over a finite set of symbols $T$ and $Q(=q(X))$ be a goal in $\boldsymbol{P}$.

(i) A language over $T$ defined by

$$
L(\boldsymbol{P}, Q, T)=\left\{w \text { in } T^{*} \mid \boldsymbol{P} \text { succeeds on } q(w)\right\}
$$

is called the success language of $Q$ in $\boldsymbol{P}$. In this case $L(\boldsymbol{P}, Q, T)$ is often denoted by $L(\boldsymbol{P}, q, T)$. If $\boldsymbol{P}$ is dominated by $p(X)$ or a program " $\boldsymbol{P}$ " is named after the goal name " $p$ " then we simply write $L(\boldsymbol{P}, T)$ and call it the success language of $\boldsymbol{P}$.

Futher,

(ii) a logic program $\boldsymbol{P}$ is called $X$ if $L(\boldsymbol{P}, Q, T)$ is an $X$ language for all goal $Q$ in $\boldsymbol{P}$.

(iii) Let $p(X, Y)$ be a goal dominating $\boldsymbol{P}$, and for $x$ in $T^{*}$, let $f_{\boldsymbol{P}}(x)=\left\{y\right.$ in $T^{*} \mid \boldsymbol{P}$ succeeds in $p(\boldsymbol{x}, \boldsymbol{y})\}$. Then, a logic progam $\boldsymbol{P}$ is called

(1) homomorphism if $f_{\boldsymbol{P}}$ is a homomorphism,

(2) (weak) coding if $f_{\boldsymbol{P}}$ is a (weak) coding,

(3) weak identity if $f_{\boldsymbol{P}}$ is a weak identity, on $T^{*}$.

Finally,

(iv) let $\boldsymbol{P}$ and $\boldsymbol{P}^{\prime}$ be two logic programs over $T$, and let $p(x)$ and $p^{\prime}(X)$ be goals in $\boldsymbol{P}, \boldsymbol{P}^{\prime}$, respectively. Then $\boldsymbol{P}$ with $p(x)$ and $\boldsymbol{P}^{\prime}$ with $p^{\prime}(X)$ are equivalent if $L(\boldsymbol{P}, p, T)$ $=L\left(\boldsymbol{P}^{\prime}, p^{\prime}, T\right)$.

We end this section with presenting a result showing the expressive capability of logic programs we are dealing with in this paper.

It has been shown in literature (e.g. [12], [13]) that for any recursively enumerable language $L$ over $T$, there exist a logic program $\boldsymbol{P}$ over $T$ and a goal $Q$ such that $L$ is the success language of $Q$ in $\boldsymbol{P}$. Conversely, it is shown that for any logic program $\boldsymbol{P}$ over $T$ and a goal $Q$, the success language $L(\boldsymbol{P}, Q, T)$ is a recursively enumerable language, which is proved by constructing a Turing machine simulating the resolution process for $Q$ from $\boldsymbol{P}$ and accepting the success language of $Q$ in $\boldsymbol{P}$ ([11]). (Note that a language is recursively enumerable if and only if it is accepted by a Turing machine.)

Hence, we have the following: 
THEOREM 2.1 The class of success languages of logic programs is equal to the class of recursively enomerable languages.

It may be possible to state that the success language of a logic program provides us a kind of model-theoretic semantics (or denotional semantics) for logic programs.

\section{Representation Theorems}

In this section several representation theorems for logic programs are presented. Most of them are easily obtained from the corresponding results in formal language theory

\subsection{Generator programs for logic programs}

We shall show that there exists a fixed logic program from which for any logic program an equivalent logic program can be obtained in terms of the composition of simpler programs. Such a fixed logic program may be called generator program.

[1] Generator Program $\boldsymbol{R}_{0}$

First we shall show that there exists a fixed simple program which plays a role of generator for the class of logic programs. Such a program can be obtained by making a slight modification to "reverse" program.

LEMMA 3.1 For any recursively enumerable language $L$ over an alphabet $T$ there exists a simple deterministic language $S p$ on $K^{+} \tilde{K}^{+}$(for some alphabet $K$ including $T$ ), and a weak identity $h$ such that $L=h\left(\left\{w \tilde{w}^{R} \mid w\right.\right.$ in $\left.\left.K^{+}\right\} \cap S p\right)$, where $S p=\left\{x \notin \tilde{\phi} \tilde{y} \tilde{y}^{R} \mid f(x)=y\right\}$, $f$ is a dgsm mapping of $A=\left(Q, K, D, d, q_{0}, F\right)$ depending $L, h$ onpreserves the alphabet of $L$ and erases other symbolds.

(See Theorem 11 in [4])

THEOREM 3.1 (Representation Theorem 1) Let $T$ be a fixed alphabet. Then, there exists a fixed logic program $\boldsymbol{R}_{0}$ with the property that for any logic program $\boldsymbol{P}$ over $T$ with a goal $p(X)$ one can find an equivalent logic program $\boldsymbol{P}^{\prime}$ with a goal $p^{\prime}(X)$ such that it can be expressed by

$$
p^{\prime}(X) \leftarrow r_{0}(X, Y), s_{P}(Y)
$$

for some simple deterministic program $\boldsymbol{S}_{\boldsymbol{P}}$.

PROOF. From Theorem 2.1 and Lemma 3.1, for any logic program $\boldsymbol{P}$ over $T$ with a goal $p(X)$ there is a simple deterministic language $S p$ on $K^{+} \widetilde{K}$ and a weak identity $h$ such that $L(\boldsymbol{P}, p, T)=h\left(\left\{w \tilde{w}^{R} \mid w\right.\right.$ in $\left.\left.K^{+}\right\} \cap S p\right)$, where $S p=\left\{x \not \tilde{\phi} \tilde{y}^{R} \mid f(x)=y\right\}, f$ is a dgsm mapping of $A=\left(Q, K, D, d, q_{0}, F\right)$ depending on $L(\boldsymbol{P}, p, T)$, and $h(a)=a$ (for all $a$ in $T$ ), $h(a)=e$ (otherwise).

Construct three logic programs so that $\boldsymbol{M}_{\boldsymbol{T}}, \boldsymbol{I}_{\boldsymbol{T}}$ and $\boldsymbol{S}_{\boldsymbol{P}}$ may determine the language $M_{0}\left(=\left\{w \tilde{w}^{R} \mid w\right.\right.$ in $\left.\left.K^{+}\right\}\right), h$, and $S p$, respectively.

(1) $\boldsymbol{M}_{T}$ is defined as follows:

$$
\begin{aligned}
& m_{T}(X) \leftarrow m 1\left(s_{1}, X,[]\right) \\
& m 1\left(s_{1},[a \mid X], Y\right) \leftarrow m 1\left(s_{1}, X,[a \mid Y]\right) \quad(\text { for all } a \text { in } K) \\
& m 1\left(s_{1},[\tilde{a} \mid X], Y\right) \leftarrow m 1\left(s_{2},[\tilde{a} \mid X], Y\right) \quad(\text { for all } a \text { in } K) \\
& m 1\left(s_{2},[],[]\right)
\end{aligned}
$$




$$
m 1\left(s_{2},[\tilde{a} \mid X],[a \mid Y]\right) \leftarrow m 1\left(s_{2}, X, Y\right) \quad(\text { for all } a \text { in } K)
$$

Clearly $\boldsymbol{M}_{T}$ determines the mirror image language, i.e., $L\left(\boldsymbol{M}_{T}, K \cup \tilde{K}\right)=\left\{w \tilde{w}^{R} \mid w\right.$ in $\left.K^{+}\right\}$. (2) $\boldsymbol{I}_{\boldsymbol{T}}$ is defined as follows:

$$
\begin{aligned}
& i_{T}([],[]) \\
& i_{T}([a \mid X],[a \mid Y]) \leftarrow i_{T}(X, Y) \quad(\text { for all } a \text { in } T) \\
& i_{T}(X,[a \mid Y]) \leftarrow i_{T}(X, Y) \quad(\text { for all } a \text { not in } T)
\end{aligned}
$$

$\boldsymbol{I}_{\boldsymbol{T}}$ is a simple projection mapping which preserves symbols from $T$ and erases others.

(3) $\boldsymbol{S}_{\boldsymbol{P}}$ is defined as follows:

$$
\begin{array}{ll}
s_{P}(X) \leftarrow s 1\left(q_{1}, X,[]\right) & \\
s 1\left(q_{1},[a \mid X], Y\right) \leftarrow s 1\left(q_{1}, X,[a \mid Y]\right) & (\text { for all } a \text { in } K \cup\{\phi\}) \\
s 1\left(q_{1},[\tilde{\phi} \mid X],[\not \mid Y]\right) \leftarrow s_{1}\left(q_{f}, X, Y\right) & \left(\text { for all } q_{f} \text { in } F\right) \\
s 1\left(q_{0},[],[]\right) & \\
s 1\left(q,\left[\tilde{w}^{R} \mid X\right],[a \mid Y]\right) \leftarrow s 1(p, X, Y) & (\text { for all } d(p, a)=(q, w))
\end{array}
$$

where $A=\left(Q, K, D, d, q_{0}, F\right)$ is a $\operatorname{dgsm} A$ given in Lemma 3.1. Then, $L\left(\boldsymbol{S}_{\boldsymbol{P}}, K \cup \tilde{K} \cup\right.$ $\{\phi, \tilde{\phi}\})=\left\{x \tilde{\phi} \tilde{\bar{\phi}} \tilde{y}^{R} \mid f(x)=y, x\right.$ in $\left.K^{*}\right\}$.

Let $\boldsymbol{P}^{\prime}$ be a logic program defined by $p^{\prime}(X) \leftarrow i_{T}(X, Y), m_{T}(Y), s_{P}(Y)$. It is easily seen that for $x$ in $T^{*}, x$ is in $L(\boldsymbol{P}, p, T)$

iff there exists $y$ such that $x=h(y)$ and

$y$ is in $M_{0} \cap S p$

iff there exists $y$ such that

$\boldsymbol{I}_{\boldsymbol{T}}$ succeeds on $i_{T}(\boldsymbol{x}, \boldsymbol{y})$,

$\boldsymbol{S}_{\boldsymbol{P}}$ succeeds on $s_{p}(\boldsymbol{y})$, and

$\boldsymbol{M}_{T}$ succeeds on $m_{T}(y)$

iff $\boldsymbol{P}^{\prime}$ succeeds on $p^{\prime}(x)$.

Let $\boldsymbol{R}_{\mathbf{0}}$ be defined by $r_{0}(X, Y) \leftarrow i_{T}(X, Y), m_{T}(Y)$. (Since $T$ is fixed, $\boldsymbol{R}_{\mathbf{0}}$ is a fixed program.) Thus, $p^{\prime}(X)$ can be expressed as the desired form (3-1).

[2] Generator Program $\boldsymbol{M}_{\mathbf{0}}$

We show that a kind of "merge" program can also play a role of generator as well as the program $\boldsymbol{R}_{\mathbf{0}}$.

LEMMA 3.2 For any recursively enumerable language $L$ over an alphabet $T$ there exist a weak identity $h$ and a regular language $R$ such that $L=h$ (shuffle $(K) \cap R)$, where $K$ is some alphabet including $T$, shuffle $(K)=\left\{x_{1} \tilde{y}_{1} \cdots x_{n} \tilde{y}_{n} \mid x_{1} \cdots x_{n}=y_{1} \cdots y_{n}\right.$ in $\left.K^{*}\right\}, R=$ $f\left(K^{*}\right), f$ is a mapping induced by a dgsm $B=\left(Q, K, K \cup \bar{D}, d^{\prime}, q_{0}, F\right)$ defined by a dgsm $A=\left(Q, K, D, d, q_{0}, F\right)$ depending on $L, d^{\prime}(q, a)=(p, a \tilde{w})$ iff $d(q, a)=(p, w), h$ preserves the alphabet $T$ and erases other symbols.

(See Theorem 13 in [4])

THEOREM 3.2 (Representation Theorem 2) Let $T$ be a fixed alphabet. Then, there 
exists a fixed program $\boldsymbol{M}_{\mathbf{0}}$ with the property that for any logic program $\boldsymbol{P}$ over $T$ with a goal $p(X)$ one can find an equivalent logic program $\boldsymbol{P}^{\prime}$ with $p^{\prime}(X)$ such that it can be expressed by

$$
p^{\prime}(X) \leftarrow m_{0}(X, Y), r_{p}(Y)
$$

for some regular program $\boldsymbol{R}_{\boldsymbol{P}}$.

PROOF * Analogous to the proof of Therem 3.1, it suffices to show that the following three logic programs satisfy the condition stated in Lemma 3.2.

(1) $\boldsymbol{M} \boldsymbol{E}_{T}$ is defined by

$$
\begin{aligned}
& m e_{T}(X) \leftarrow m e 1(X,[],[]) \\
& m e 1([], X, X) \\
& m e 1([\tilde{a} \mid X] Y, Z) \leftarrow m e 1(X, Y,[a \mid Z]) \quad \text { (for all } a \text { in } K) \\
& m e 1([a \mid X], Y, Z) \leftarrow m e 1(X,[a \mid Y], Z) \quad \text { (for all } a \text { in } K) .
\end{aligned}
$$

$\boldsymbol{M} \boldsymbol{E}_{\boldsymbol{T}}$ determines what is called the twin shuffle language, i.e.,

$$
L\left(\boldsymbol{M} \boldsymbol{E}_{\boldsymbol{T}}, T \cup \bar{T}\right)=\left\{x_{1} \tilde{y}_{1} \cdots x_{n} \tilde{y}_{n} \mid x_{1} \cdots x_{n}=y_{1} \cdots y_{n} \text { in } K^{*}\right\} .
$$

(2) $\boldsymbol{I}_{T}$ is the same as the one defined above in the proof for Theorem 3.1.

(3) $\boldsymbol{R}_{\boldsymbol{P}}$ is defined:

Let $A=\left(Q, K, D, d, p_{0}, F\right)$ be a given dgsm in Lemma 3.2 .

$$
\begin{aligned}
& r_{p}(X) \leftarrow r 1\left(p_{0}, X,[]\right) \\
& r 1(p,[a \mid X], Y) \leftarrow r 1\left(p_{a}, X,[a \mid Y]\right) \quad(\text { for all } d(p, a)=(q, w)) \\
& r 1\left(p_{a},[\tilde{w}],[a \mid Y]\right) \leftarrow r 1(q, X, Y) \quad(\text { for all } d(p, a)=(q, w)) \\
& r 1\left(p_{f},[],[]\right) \quad\left(\text { for all } p_{f} \text { in } F\right) \\
& L\left(\boldsymbol{R}_{p}, K \cup \tilde{K}\right)=\left\{a_{1} \tilde{w}_{1} \cdots a_{n} \tilde{w}_{n} \mid d\left(p_{0}, a_{1} \cdots a_{n}\right)=\left(q, w_{1} \cdots w_{n}\right), q \text { in } F\right\}=f\left(K^{*}\right) .
\end{aligned}
$$

Let $\boldsymbol{M}_{\mathbf{0}}$ be defined by

$$
m_{0}(X, Y) \leftarrow i_{T}(X, Y), m e_{T}(Y) .
$$

(Again since $T$ is fixed, $\boldsymbol{M}_{\mathbf{0}}$ is a fixed program.) Further, let $\boldsymbol{P}^{\prime}$ be defined by $p^{\prime}(X)$ $\leftarrow m_{0}(X, Y), r_{p}(Y)$. To complete the proof it suffices to check if the following relation holds: for $x$ in $T^{*}, \boldsymbol{P}^{\prime}$ succeeds on $p^{\prime}(x)$ iff $x$ is in $L(\boldsymbol{P}, p, T)$.

[3] Generator Program $\boldsymbol{D}_{\mathbf{0}}$

It is demonstrated that a program which behaves as a checker for well-pairedness can be a generator for the class of logic programs.

LEMMA 3.3 For any recursively enumerable language $L$ over $T$, there exist a linear grammar $G_{L}=\left(\{S\}, T^{\prime} \cup \tilde{T}^{\prime}, P_{L}, S\right)$ and a weak coding $h$ satisfying the following properties that

(i) $L=h\left(D_{r} \cap L\left(G_{L}\right)\right)$,

(ii) $T$ is a subset of $T^{\prime}$, and $h(a)=a$ (for all $a$ in $T$ ), 


$$
h(a)=e \quad\left(\text { for all } a \text { in } T^{\prime} \cup \tilde{T}^{\prime}-T\right),
$$

(iii) $P_{L}=\left\{S \rightarrow u_{i} S v_{i} \mid 1 \leq i \leq n\right\} \cap\{S \rightarrow w\}$, where $w, u_{i}, v_{i}(1 \leq i \leq n)$ are in $\left(T^{\prime} \cup \widetilde{T}^{\prime}\right)^{*}, D_{r}$ is the Dyck language over $T^{\prime}\left(r\right.$ : the cardinality of $\left.T^{\prime}\right), \widetilde{T}^{\prime}=\left\{\tilde{a} \mid a\right.$ in $\left.T^{\prime}\right\}$.

(See [7] for the proof.)

\section{Important Remarks.}

(1) A linear grammar $G_{L}$, which is called a minimal linear grammar ([2]), depends on $L$, while $h$ depends on only $T$.

(2) A careful and patient observation of the proof for Lemma 3.3 in [7] leads to the fact that by making a slight modification one can obtain another $G_{L}$ with its additional property, that is,

(iv) none of the two among $\boldsymbol{w}, u_{i}, v_{i}(1 \leq i \leq n)$ is identical, each of them is nonempty and $\boldsymbol{w}$ does not depend on $L$.

THEOREM 3.3 (Representation Theorem 3) Let $T$ be a fixed alphabet. Then, there exists a fixed program $\boldsymbol{D}_{\mathbf{0}}$ with the property that for any logic program $\boldsymbol{P}$ over $T$ with a goal $p(X)$ one can find an equivalent logic program $\boldsymbol{P}^{\prime}$ with a goal $p^{\prime}(X)$ such that it can be expressed by

$$
p^{\prime}(X) \leftarrow d_{0}(X, Y), \operatorname{lin}_{p}(Y)
$$

for some linear program $\boldsymbol{L I} \boldsymbol{N}_{\boldsymbol{P}}$.

PROOF. From Theorem 2.1 and Lemma 3.3 for any logic program $P$ over $T$ and a goal $p(X)$ there exist a homomorphism $h$ from $\left(T^{\prime} \cup \tilde{T}^{\prime}\right)^{*}$ to $T^{*}$ and a linear grammar $G_{L}$ with the property described above, and that $x$ is in $L(\boldsymbol{P}, p, T)$ iff there is $y$ such that $h(y)=x$ and $y$ is in $D_{r} \cap L\left(G_{L}\right)$, where $r$ is the cardinality of $T^{\prime}$.

Construct two logic programs $\boldsymbol{D}_{T}, \boldsymbol{L} \boldsymbol{I} \boldsymbol{N}_{P}$ so that it may hold that (i) $L\left(\boldsymbol{D}_{T}, T^{\prime} \cup \widetilde{T}\right)$ $=D_{r}$, and (ii) $L\left(\boldsymbol{L I} \boldsymbol{N}_{P}, T^{\prime} \cup \widetilde{T}^{\prime}\right)=L\left(G_{L}\right)$ :

(1) $d_{T}(X) \leftarrow d y c k(X,[])$

$\operatorname{dyck}([],[])$

$d y c k([a \mid X], Y) \leftarrow d y c k(X,[a \mid Y]) \quad\left(\right.$ for all $a$ in $\left.T^{\prime}\right)$

$d y c k([\tilde{a} \mid X],[a \mid Y]) \leftarrow d y c k(X, Y) \quad\left(\right.$ for all $a$ in $\left.T^{\prime}\right)$

(2) $\operatorname{lin}_{p}(X) \leftarrow \operatorname{lin}\left(p_{1}, X,[]\right)$

$\operatorname{lin}\left(p_{1},[w \mid X], Y\right) \leftarrow \operatorname{lin}\left(p_{2}, X, Y\right) \quad\left(w\right.$ is the word such that $S \rightarrow w$ in $\left.P_{L}\right)$

$\operatorname{lin}\left(p_{2},[],[]\right)$

$\operatorname{lin}\left(p_{1},\left[u_{i} \mid X\right], Y\right) \leftarrow \operatorname{lin}\left(p_{1}, X,\left[u_{i} \mid Y\right]\right) \quad\left(\right.$ for all $S \rightarrow u_{i} S v_{i}$ in $P_{L}$ of $G_{L}$ )

$\operatorname{lin}\left(p_{2},\left[v_{i} \mid X\right],\left[u_{i} \mid Y\right]\right) \leftarrow \operatorname{lin}\left(p_{2}, X, Y\right) \quad\left(\right.$ for all $S \rightarrow u_{i} S v_{i}$ in $P_{L}$ of $G_{L}$ ).

Since it is almost obvious that $L\left(\boldsymbol{D}_{\boldsymbol{T}}, T^{\prime} \cup \tilde{T}^{\prime}\right)=D_{r}$, we shall check that $L\left(\boldsymbol{L} \boldsymbol{I} \boldsymbol{N}_{\boldsymbol{P}}\right.$, $\left.T^{\prime} \cup \tilde{T}^{\prime}\right)=L\left(G_{L}\right)$. For any $x$ in $L\left(G_{L}\right)$, there is a sequence of production rules $r_{1}, \cdots$, $r_{k}, r_{0}$ such that

$$
x=u_{i 1} \cdots u_{i k} w v_{i k} \cdots v_{i 1}, r_{j}: S \rightarrow u_{i j} S v_{i j}(1 \leq j \leq k) \quad \text { and } r_{0}: S \rightarrow w .
$$


Let $x=x_{1} w x_{2}$, where $x_{1}=u_{i 1} \cdots u_{i k}, x_{2}=v_{i k_{*}}{ }^{*} \cdots v_{i 1}$, then we have that $\operatorname{lin}_{p}(\boldsymbol{x})$ succeeds if $\operatorname{lin}\left(p_{1}\right.$, $\boldsymbol{x}$, [ ] ) succeeds, and that $\operatorname{lin}\left(p_{1}, \boldsymbol{x}_{1} \boldsymbol{w} \boldsymbol{x}_{2},[]\right.$ ) succeeds if $\operatorname{lin}\left(p_{1}, \boldsymbol{w} \boldsymbol{x}_{2}, \boldsymbol{f}\left(\boldsymbol{x}_{1}\right)\right)$ succeeds, if $\operatorname{lin}\left(p_{2}, \boldsymbol{x}_{2}, \boldsymbol{f}\left(\boldsymbol{x}_{1}\right)\right)$ succeeds, if $\operatorname{lin}\left(p_{2},[],[]\right)$ succeeds, where $f$ is defined by $f(e)=e$, $f\left(u_{j} x\right)=f(x) f\left(u_{j}\right)$ for $u_{j}$ in $\left\{u_{1}, \cdots, u_{n}\right\}, x$ in $\left\{u_{1}, \cdots, u_{n}\right\}^{*}$.

Thus, we eventually have $\operatorname{lin}_{p}(\boldsymbol{x})$ succeeds. The converse relation is straightforwardly proved. Hence, it is obtained that $L\left(\boldsymbol{L} \boldsymbol{I} \boldsymbol{N}_{P}, T^{\prime} \cup \tilde{T}^{\prime}\right)=L\left(G_{L}\right)$.

Now, let $\boldsymbol{D}_{0}$ be defined by $d_{0}(X, Y) \leftarrow i_{T}(X, Y), d_{T}(Y)$, where $i_{T}(X, Y)$ is a predicate already appeared in Theorem 3.1 and Theorem 3.2. (Since $T$ is fixed, so is $\boldsymbol{D}_{0}$.) $F$ urther let $p^{\prime}(X) \leftarrow d_{0}(X, Y), \operatorname{lin}_{p}(Y)$. To complete the proof, we have only to show that $p^{\prime}(x)$ succeeds iff $x$ is in $L(P, p, T)$, and this is easily checked in the following way:

$p^{\prime}(x)$ succeeds iff there is $y$ such that $i_{T}(\boldsymbol{x}, \boldsymbol{y}), d_{T}(\boldsymbol{y})$, and $\operatorname{lin}_{p}(\boldsymbol{y})$ succeed

iff there is $y$ such that $h(y)=x, y$ in $L\left(\boldsymbol{D}_{T}, T^{\prime} \cup \tilde{T}^{\prime}\right)$, and $y$ in $L\left(G_{L}\right)$

iff $x$ is in $L(\boldsymbol{P}, p, T)$.

REMARK (i) A program $\boldsymbol{D}_{T}$ whose success language is a Dyck language works for checking "well-pairedness" of an input string in $\boldsymbol{D}_{0}$.

(ii) A program structure of $\boldsymbol{L} \boldsymbol{I} \boldsymbol{N}_{P}$ is quite similar to that of $\boldsymbol{S}_{\boldsymbol{P}}$ in Theorem 3.1.

Later we will discuss the close relationships among these generator programs and what operations are really primitive for expressing logic programs.

\subsection{Decomposing logic programs}

As we have seen in the previous subsection, a logic program can be expressed as a conjunctive formula comprising a simpler program consisting of two components. Further, one of the two is quite simpler than the other in that it just works as a simple homomorphism (actually, a weak identity mapping).

We shall show a representation theorem for logic programs in which for any logic program one can find an equivalent logic program expressed as a conjuntive formula of two fixed programs and three simple homomorphism programs. Exactly, one of the three can be fixed.

LEMMA 3.4 For any simple deterministic (context-free) language $L$, there exist a coding $f$ and a homomorphism $h$ such that $L=f\left(h^{-1}\left(\varnothing D_{2}\right)\right)$, where $D_{2}$ is a Dyck language, $\phi$ is a (new) symbol.

(The way of the proof for Lemma 3.4 is similar to that of the proof for the main theorem in [5]. See Appendix for the proof.)

This lemma leads to another representation for logic programs which may be called "decomposition theorem" for logic programs.

THEOREM 3.4 (Representation Theorem 4) Let $T$ be a fixed alphabet. Then, there exist fixed logic programs $\boldsymbol{I}, \boldsymbol{D}$ and $\boldsymbol{M}$ with the property that for any logic program $\boldsymbol{P}$ over $T$ with a goal $p(X)$ one can find an equivalent logic program $\boldsymbol{P}^{\prime}$ with a goal $p^{\prime}(X)$ such that it can be expressed by

$$
p^{\prime}(X) \leftarrow i(X, Y), m(Y), f_{p}(Y, V), h_{p}(V, Z), d(Z)
$$


for some coding program $\boldsymbol{F}_{\boldsymbol{P}}$ and a homomorphism program $\boldsymbol{H}_{\boldsymbol{P}}$.

PRoof. From the proof of Theorem 3.1 there exist fixed programs $\boldsymbol{M}_{\boldsymbol{T}}$ and $\boldsymbol{I}_{\boldsymbol{T}}$ such that for a given logic program $\boldsymbol{P}$ with a goal $p(X)$ one can have an equivalent logic program $\boldsymbol{P}^{\prime}$ with a goal $p^{\prime}(X)$ such that it is expressed by $p^{\prime}(X) \leftarrow i_{T}(X, Y)$, $s_{p}(Y)$, for some simple deterministic program $\boldsymbol{S}_{\boldsymbol{P}}$. Further, Lemma 3.4 tells that there exist a coding $f_{L}$ and a homomorphism $h_{L}$ such that $L\left(=L\left(\boldsymbol{S}_{\boldsymbol{P}}, K \cup \widetilde{K}\right)\right)=f_{L}\left(h_{L}^{-1}\left(\phi D_{2}\right)\right)$. Let $\boldsymbol{I}$ be $\boldsymbol{I}_{\boldsymbol{T}}$ and $\boldsymbol{M}$ be $\boldsymbol{M}_{\boldsymbol{T}}$. Then, it suffices to show that one can construct a coding program $\boldsymbol{F}_{\boldsymbol{P}}$, a homomorphism program $\boldsymbol{H}_{\boldsymbol{P}}$ and a fixed program $\boldsymbol{D}$ such that (i) $\boldsymbol{D}$ determines the Dyck language $\phi D_{2}$ and (ii) $s_{p}(Y)$ can be expressed as a conjunctive formula of $f_{p}(Y, V), h_{p}(V, Z)$ and $d(Z)$.

Define $\boldsymbol{F}_{P}, \boldsymbol{H}_{\boldsymbol{P}}$ and $\boldsymbol{D}$ as follows :

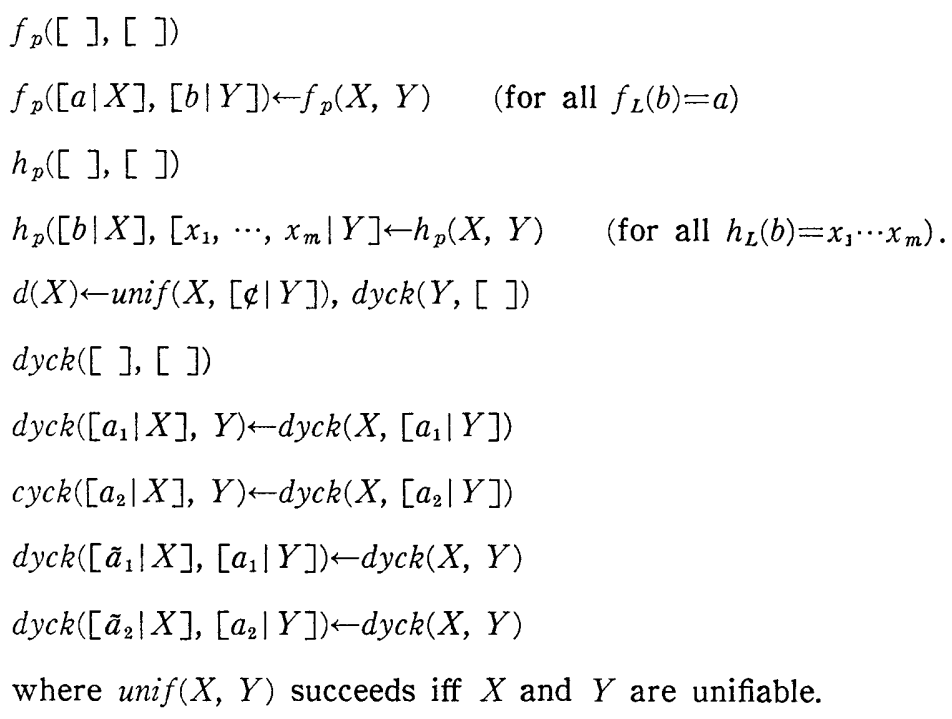

Clearly, $L\left(\boldsymbol{D},\left\{a_{1}, a_{2}, \tilde{a}_{1}, \tilde{a}_{2}, \phi\right\}\right)=\varnothing D_{2}$, and it is easily seen that $\boldsymbol{S}_{\boldsymbol{P}}$ succeeds on $s_{p}(\boldsymbol{y})$

iff $y$ is in $L\left(\boldsymbol{S}_{\boldsymbol{P}}, K \cup \tilde{K}\right)$

iff there exist $v$ and $z$ such that $f_{L}(v)=y, h_{L}(v)=z$

iff there exist $v$ and $z$ such that

$$
\begin{aligned}
& \boldsymbol{F}_{\boldsymbol{P}} \text { succeeds on } f_{p}(\boldsymbol{y}, \boldsymbol{v}), \\
& \boldsymbol{H}_{\boldsymbol{P}} \text { succeeds on } h_{p}(\boldsymbol{v}, \boldsymbol{z}) \text {, and } \\
& \boldsymbol{D} \text { succeeds on } d(\boldsymbol{z}) .
\end{aligned}
$$

This implies that $s_{P}(Y) \leftarrow f_{P}(Y, V), h_{p}(V, Z), d(Z)$. :Thus, eventually, we have that $p^{\prime}(X) \leftarrow(X, Y), m(Y), f_{P}(Y, V), h_{P}(V, Z), d(Z)$. This completes the proof.

REMARK. The teaching of Theorem 3.4 is that using two fixed logic programs $\boldsymbol{M}$ (a modified "reverse" program) and $\boldsymbol{D}$ (a "checking well-pairedness" program) any logic program $\boldsymbol{P}$ can be reducible into three homomorphism $\boldsymbol{I}, \boldsymbol{F}_{\boldsymbol{P}}$ and $\boldsymbol{H}_{\boldsymbol{P}}$ that have a very simple structure. 


\section{What are Primitives?}

We have seen in Section 3 that several specific types of logic programs can play a significant role as a generator in expressing logic programs. In this section we shall discuss this issue on generator in more detail.

\subsection{Primitives for generators}

Getting back to the representation theorems, a generator program $\boldsymbol{R}_{0}$ in (3-1) of Theorem 3.1 was constructed from a weak identity program $\boldsymbol{I}_{\boldsymbol{T}}$ and a logic program $M_{T}$, i.e.,

where

$$
r_{0}(X) \leftarrow i_{T}(X, Y), m_{T}(Y)
$$

[0] $i_{T}([],[])$

$i_{T}([a \mid X),[a \mid Y]) \leftarrow i_{T}(X, Y) \quad($ for all $a$ in $T)$

$i_{T}(X,[a \mid Y]) \leftarrow i_{T}(X, Y) \quad$ (for all $a$ not in $T$ ), and

we observe that $m_{T}(X)$ can be re-defined as follows:

[1] $m_{T}(X) \leftarrow a p p e n d(Y, Z, X), \operatorname{cop} y\left(Y, Y^{\prime}\right), \operatorname{reverse}\left(Y^{\prime}, Z\right)$

$\operatorname{copy}([],[])$

$\operatorname{cop} y([a \mid X],[\tilde{a} \mid Y]) \leftarrow \operatorname{cop} y(X, Y) \quad($ for all $a$ in $K)$

reverse $([],[])$

reverse $([X \mid Y], Z) \leftarrow \operatorname{reverse}(Y, T)$, append $(T,[X], Z)$.

Similarly, from an observrtion of a generator program $\boldsymbol{M}_{0}$ in (3-2) of Theorem 3.2 we have:

where

$$
m_{0}(X) \leftarrow i_{T}(X, Y), m e_{T}(Y)
$$

[2] $m e_{T}(X) \leftarrow \operatorname{merge}(Y, Z, X), \operatorname{copy}(Y, Z)$

$\operatorname{merge}(X,[], X)$

$\operatorname{merge}([], X, X)$

$\operatorname{merge}([a \mid X], Y,[a \mid Z]) \leftarrow \operatorname{merge}(X, Y, Z) \quad($ for all $a$ in $K)$

$\operatorname{merge}(X,[\tilde{a} \mid Y],[\tilde{a} \mid Z]) \leftarrow \operatorname{merge}(X, Y, Z) \quad($ for all $a$ in $K$ )

Further, a generator program $\boldsymbol{D}_{0}$ in (3-3) of Theorem 3.3 is analysed as follows:

where

$$
d_{0}(X) \leftarrow i_{T}(X, Y), d_{T}(Y)
$$

[3] $d_{T}(X) \leftarrow-d y c k(X,[])$

$d y c k([],[])$ 


$$
\begin{aligned}
& d y c k\left([ a | X ) \leftarrow d y c k ( X , [ a | Y ] ) \quad \left(\text { for all } a \text { in } T^{\prime}\right.\right. \text { ) } \\
& d y c k([\tilde{a} \mid X],[a \mid Y]) \leftarrow d y c k(X, Y) \quad\left(\text { for all } a \text { in } T^{\prime}\right) .
\end{aligned}
$$

It is easily seen that each generator contains a common homomorphism (exactly weakidentity) program $\boldsymbol{I}_{\boldsymbol{T}}$ which serves as a kind of "filter". That means the essentially unique parts of generator programs are $m_{T}(X), m e_{T}(X)$ and $d_{T}(X)$.

Thus, it is possible to say that "append", "copy", "merge", "dyck" are all primitives for a generator program in the representation theorem. However, noting that "copy" is a special type of a homomorphism program and "append" and "dyck" are restricted versions of "merge", we may conclude that the filtering function ("homomorphism") and the merging function ("merge") are fully primitive for expressing logic programs.

\subsection{Extended reverse programs}

We shall show there exists a type of logic program which can take the place of various basic programs appearing in the representation results.

Let $f$ be a mapping from $T^{*}$ to $K^{*}$. Then, consider a logic program dominated by a predicate " $(f)$-reverse $(X, Y)$ ", which is defined by $(f)$-reverse $(\boldsymbol{x}, \boldsymbol{y})$ succeeds iff so does reverse $(\boldsymbol{f}(\boldsymbol{x}), \boldsymbol{y})$. We call this extended reverse program. (Notice that if $f$ is an identity, then $(f)$-reverse $(X, Y)$ is an ordinary "reverse" predicate.)

ExAmple 1. Let $f$ be defined by $f(a)=\tilde{a}, f(b)=\tilde{b}, f(c)=\tilde{c}$. Then, $(f)$-reverse $(X, Y)$ may be, for example, defined as follows:

$$
\begin{aligned}
& (f)-\operatorname{reverse}(X, Y) \leftarrow \operatorname{rev}(X,[], Y) \\
& \operatorname{rev}([], X, X) \\
& \operatorname{rev}([a \mid X], Y, Z) \leftarrow \operatorname{rev}(X,[\tilde{a} \mid Y], Z) \\
& \operatorname{rev}([b \mid X], Y, Z) \leftarrow \operatorname{rev}(X,[\tilde{b} \mid Y[, Z) \\
& \operatorname{rev}([c \mid X], Y, Z) \leftarrow \operatorname{rev}(X,[\tilde{c} \mid Y], Z) .
\end{aligned}
$$

Let $p(X) \leftarrow a p p e n d(Y, Z, X),(f)$-reverse $(Y, Z)$, then the success language of this program $\left\{w \tilde{w}^{R} \mid w\right.$ in $\left.\{a, b, c\}^{*}\right\}$ is context-free.

Now, let us see the next one.

Example 2. Let $f$ be a mapping defined by $f(x)=\tilde{x}^{R}$, for all $x$ in $T^{*}$. Then, it is seen that

$(f)$-reverse $(\boldsymbol{x}, \boldsymbol{y})$ succeeds iff reverse $(\boldsymbol{f}(\boldsymbol{x}), \boldsymbol{y})$ succeeds

iff reverse $\left(\tilde{\boldsymbol{x}}^{R}, \boldsymbol{y}\right)$ succeeds

iff $\tilde{x}=y$.

Let $\boldsymbol{P}$ be a program dominated by $p(X) \leftarrow \operatorname{append}(Y, Z, X),(f)$-reverse $(Y, Z)$. Then, the success language $L(\boldsymbol{P}, T \cup \tilde{T})$ is $\left\{w \tilde{w} \mid w\right.$ in $\left.T^{*}\right\}$ which is context-sensitive.

Thus, $(f)$-reverse can define a number of different classes of logic programs by varing a mapping $f$.

Now we wish to call back one's attention to the representation theorems. In the representation formula (3-1) of Theorem 3.1 a logic program can be expressed by 
$p(X) \leftarrow r_{0}(X, Y), s_{P}(Y)$, where

(0) $r_{0}(X, Y \leftarrow) i_{T}(X, Y), m_{T}(Y)$

(1) $s_{P}\left(X \leftarrow s 1\left(q_{1}, X,[]\right)\right.$

$s 1\left(q_{1},[a \mid X], Y\right) \leftarrow s 1\left(q_{1}, X,[a \mid Y]\right) \quad($ for all $a$ in $K \cup\{\not \subset\})$

$s 1\left(q_{1},[\tilde{\phi} \mid X],[\phi \mid Y]\right) \leftarrow s 1\left(\left(q_{f}, X, Y\right) \quad\right.$ (for all $q_{f}$ in $\left.F\right)$

$\operatorname{si}\left(q_{0},[],[]\right)$

(2) $s 1\left(q,\left[\tilde{w}^{R} \mid X\right],[a \mid Y]\right) \leftarrow s 1(p, X, Y) \quad($ for all $d(p, a)=(q, w))$

where $A=\left(Q, K, D, d, q_{0}, F\right)$ is a dgsm.

Let $f_{T}$ be defined by $f_{T}(a)=\tilde{a}$ (for all $a$ in $T$ ). Then, it is easily seen that

$$
m_{T}(X) \leftarrow \text { append }(Y, Z, X),\left(f_{T}\right) \text {-reverse }(Y, Z) \cdots\left(F_{1}\right) \text {. }
$$

Further, letting $f_{P}$ be a mapping defined by $f_{P}(x)=f(x)$, where $f$ is a dgsm mapping induced by $A$, then we have

$$
s_{P}(X) \leftarrow \operatorname{append}(Y, Z, X),\left(f_{P}\right) \text {-reverse }(Y, Z) \cdots\left(F_{2}\right) .
$$

Recall the representation formula (3-3) of Theorem 3.3 in which a logic program can be expressed by

where

$$
p(X) \leftarrow d_{0}(X, Y), \operatorname{lin}_{P}(Y)
$$

(3) $\operatorname{lin}_{P}(X) \leftarrow \operatorname{lin}\left(p_{1}, X,[]\right)$

for each rule $S \rightarrow u S v$ in $P_{L}$ of $G_{L}$,

$\operatorname{lin}\left(p_{1},[u \mid X], Y\right) \leftarrow \operatorname{lin}\left(p_{1}, X,[u \mid Y]\right)$

$\operatorname{lin}\left(p_{1},[w \mid X], Y\right) \leftarrow \operatorname{lin}\left(p_{2}, X, Y\right) \quad\left(S \rightarrow w\right.$ in $\left.P_{L}\right)$

$\operatorname{lin}\left(p_{2},[],[]\right)$

$\operatorname{lin}\left(p_{2},[v \mid X],[u \mid Y]\right) \leftarrow \operatorname{lin}\left(p_{2}, X, Y\right)$.

$G_{L}=\left(\{S\}, T^{\prime}, P_{L}, S\right), P_{L}=\left\{S \rightarrow u_{1} S v_{1}, \cdots, S \rightarrow u_{n} S v_{n}, S \rightarrow w\right\}$.

Let $f$ be defined as follows:

$$
\begin{aligned}
& f(u)=v^{R} \text { for all } S \rightarrow u S v \text { in } P_{L} \\
& f\left(u u^{\prime}\right)=f(u) f\left(u^{\prime}\right) \text { for all } u, u^{\prime} \text { in }\left\{u_{1}, \cdots, u_{n}\right\}^{*}
\end{aligned}
$$

Here we claim that

$\operatorname{lin}_{P}(\boldsymbol{x})$ succeeds iff append $(\boldsymbol{y}, \boldsymbol{w} \boldsymbol{z}, \boldsymbol{x})$ and $(f)$-reverse $(\boldsymbol{y}, \boldsymbol{z})$ succeed for some $y, z$. Since $L\left(\boldsymbol{L} \boldsymbol{I} \boldsymbol{N}_{\boldsymbol{P}}, T \cup \tilde{T}^{\prime}\right)=L\left(G_{L}\right)$, which is proved in the proof of Theorem 3.3, for the purpose of verifying the claim it suffices to show that $x$ is in $L\left(G_{L}\right)$ iff append $(\boldsymbol{y}, \boldsymbol{w z}, \boldsymbol{x})$ and $(f)$-reverse $(\boldsymbol{y}, \boldsymbol{z})$ succeed for some $y, z$ in $T^{*}$. For any $x$ in $L\left(G_{L}\right)$, there is a sequence of rules $r_{1}, \cdots, r_{k}, r_{0}$ such that 


$$
x=u_{i 1} \cdots u_{i k} w v_{i k} \cdots v_{i 1}, r_{j}: S \rightarrow u_{i j} S v_{i j}(1 \leq j \leq k) \quad \text { and } r_{0}: S \rightarrow w .
$$

Hence, let $x=x_{1} w x_{2}$, where $x_{1}=u_{i 1} \cdots u_{i k}, \quad x_{2}=v_{i k} \cdots v_{i 1}$, then we have that append $\left(\boldsymbol{x}_{1}\right.$, $\left.\boldsymbol{w} \boldsymbol{x}_{2}, \boldsymbol{x}\right)$ succeeds and $(f)$-reverse $\left(\boldsymbol{x}_{1}, \boldsymbol{x}_{2}\right)$ is invoked. By the definition of $f, f\left(x_{1}\right)=$ $f\left(u_{i 1} \cdots u_{i k}\right)=v_{i 1}{ }^{R} \cdots v_{i k}{ }^{R}=\left(v_{i k} \cdots v_{i 1}\right)^{R}=x_{2}{ }^{R}$. Since $\left(f_{2}\right)$-veverse $\left(\boldsymbol{x}_{1}, \boldsymbol{x}_{2}\right)$ succeeds iff reverse $\left(\boldsymbol{f}\left(\boldsymbol{x}_{1}\right), \boldsymbol{x}_{2}\right)$ succeeds, we have that $(f)$-reverse $\left(\boldsymbol{x}_{1}, \boldsymbol{x}_{2}\right)$ succeeds. The converse relation is proved in a similar manner. Thus, we eventually have

$$
\operatorname{lin}_{p}(X) \leftarrow \operatorname{append}(Y,[w \mid Z], X),(f) \text {-reverse }(Y, Z) \cdots\left(F_{3}\right)
$$

It should be noted that for a homomorphism $h$, if one define a mapping $f_{h}$ by $f_{h}(x)=h(x)^{R}$, then $\left(f_{h}\right)$-reverse $(\boldsymbol{x}, \boldsymbol{y})$ succeeds iff $h(x)=y$. Hence, a weak identity program $\boldsymbol{I}_{\boldsymbol{T}}$ dominated by $i_{T}(X, Y)$ and involved in all representation results is expressed by

$$
i_{T}(X, Y) \leftarrow\left(f_{h}\right) \text {-reverse }(Y, X) \cdots\left(F_{4}\right)
$$

Summarizing our argument on the use of extended reverse programs for expressing various types of basic elements in the representation results, from $\left(F_{1}\right),\left(F_{2}\right),\left(F_{4}\right)$ and (3-1) we obtain another representation theorem for logic programs.

THEOREM 4.1 (Representation Theorem 5) Let $T$ be a fixed alphabet. Then, there exist mappings $f_{h}, f_{T}$ with the property that for any logic program $\boldsymbol{P}$ over $T$ with a goal $p(X)$ one can find an equivalent logic program $\boldsymbol{P}^{\prime}$ with a goal $p^{\prime}(X)$ such that it is expressed by

$$
\begin{gathered}
p^{\prime}(X) \leftarrow\left(f_{h}\right) \text {-reverse }(Y, X), \text { append }\left(Z_{1}, Z_{2}, Y\right),\left(f_{T}\right) \text {-reverse }\left(Z_{1}, Z_{2}\right), \\
\quad \text { append }\left(W_{1}, W_{2}, Y\right),\left(f_{P}\right) \text {-reverse }\left(W_{1}, W_{2}\right),
\end{gathered}
$$

for some mapping $f_{P}$.

\section{Concluding Remarks}

Through the formal language theoretic formulation, we have shown several representation theorems for logic programs. First, we introduced the concept of the success language of a logic program, and associating a logic program with its success language we gave a formal language theoretic semantics of logic programs.

Further, using the language theoretic semantics several representation theorems for logic programs were provided in which some types of fixed logic programs called generator programs play central roles in the representation.

Then, it has been considered the problem of what operation is primitive for the representation of logic programs. It was shown that the filtering function by a homomorphism and the merging function are sufficiently primitive in the sense that for any logic program one can find an equivalent logic program which is expressed within the use of combination of these two programs.

Finally, by introducing the concept of an extended reverse predicate, it has been proved that one need only "append" and "extended reverse" functions in representing logic programs.

For the future research in this direction, using a model-theoretic semantics in 
terms of the success language one may discuss many issues on the properties of a logic program such as program transformation, program classification, program synthesis, and so forth, some of those which we are about to work on.

\section{Acknowledgements}

The author is indebted to Dr. Tosio Kitagawa, the president of IIAS-SIS, Dr. Hajime Enomoto, the director of IIAS-SIS, for their useful suggestion and warm encouragement.

He is also grateful to his colleagues, Toshiro Minami, Taishin Nishida who worked through an earlier draft of the paper and suggested the present improved formulation.

This is a part of the work in the major R \& D of the Fifth Generation Computer Project, conducted under program set up by MITI.

\section{References}

[1] Colmeraurer, A., Les systemes-Q ou un formalisme pour analyser et synthetiser des ordinateur, Internal publication no. 43, Dept. d'Informatigue, University de Montreal, Canada, September, (1970).

[2] Chomsky, N. and Schutzenberger, M.P.: The algebraic Theory of context-free languages, in "Computer Programming and Formal Systems (Braffort and Hirschberg, eds.), North-Holland, Amsterdam, (1962), 118-161.

[3] van Emden, M.H. and Kowalski, R.A., The Semantics of Predicate Logic as a Programming Language, JACM 23, (1976), 733-742.

[4] Engelfriet, J. and Rozenberg, G., Fixed Point Languages, Equality Language, and Representation of Recursively Enumerable Languages, JACM 27, (1980), 299-518.

[5] Greibach, S.A., The Hardest Context-free Language, SIAM J. Computing 2, (1973), 304310.

[6] HARRISON, M.A., Introduction to Formal Language Theory, Addison-Wesley, (1978).

[7] Hirose, S., Okawa, S. and Yoneda, M., A Homomorphic Characterization of Recurable Languages, Theoretical Computer Science 35, (1985), 261-269.

[8] Hopcroft, J.E. and Ullman, J.D., Introduction to Autroduction to Automata Theory, Languages, and Computation, Addison-Wesley, (1979).

[9] Kowalski, R., Predicate logic as a programming language, In Proceedings of IFIP-74, (1974), 569-574.

[10] SalomaA, A., Formal Languages, Academic Press, 1973.

[11] Shapiro, E.Y., Alternation and the Computational Complexity of Logic Programs, J. Logic Programming 1, (1984), 19-33.

[12] Tärnlund, S.A., Horn Clause Computability, BIT 17, (1977), 215-226.

[13] Yokomori, T., A Logic Program Schema and Its Applications, In Proceedings of 9 th IJCAI, UCLA, CA, (1985), 723-725.

\section{Appendix [The proof of Lemma 3.4]}

We show the following: for any simple deterministic grammar $G$, there exist a simple deterministic grammar $G_{0}$, a coding $f$ and a homomorphism $h$ such that $L(G)=$ $f\left(L\left(G_{0}\right)\right)$ and $L\left(G_{0}\right)=h^{-1}\left(\phi D_{2}\right)$. This immediately completes the proof.

Let $G=\left(N, T, P, S_{0}\right)$ be a simple deterministic grammar such that $L=L(G)$, where $N=\left\{A_{1}\left(=S_{0}\right), \cdots, A_{n}\right\}$. We may assume that $S_{0}$ does not appear in the right-hand side 
of any rule in $P$.

Construct a simple deterministic grammar $G_{0}=\left(N, T^{\prime}, P^{\prime}, S_{0}\right)$ as follows: $T^{\prime}=\{[A$, a] $\mid A \rightarrow a x$ in $P\}, P^{\prime}=\{A \rightarrow[A, a] x \mid A \rightarrow a x$ in $P\}$. Define $f$ by

$$
f([A, a])=a \quad \text { for }[A, a] \text { in } T^{\prime} .
$$

Then, it is obvious that $G_{0}$ is simple deterministic and $L(G)=f\left(L\left(G_{0}\right)\right)$ holds.

Now, since $G$ is simple deterministic, one can define a homomorphism $h$ from $T^{*}$ * into $\left\{a_{1}, a_{2}, \tilde{a}_{1}, \tilde{a}_{2}, \not \subset\right\}^{*}$ by

$$
\begin{aligned}
& h\left(\left[A_{i}, a\right]\right)=\tilde{a}_{1} \tilde{a}_{2}{ }^{i} \tilde{a}_{1} a_{1} a_{2}{ }^{j m} a_{1} \cdots a_{1} a_{2}{ }^{j 1} a_{1}, \\
& \quad \text { if } A_{i} \rightarrow a A_{j 1} \cdots A_{j m} \text { in } P \text { and } i \neq 1, \\
& h\left(\left[A_{1}, a\right]\right)=\varnothing a_{1} a_{2}{ }^{j m} a_{1} \cdots a_{1} a_{2}{ }^{j 1} a_{1}, \\
& \quad \text { if } A_{1} \rightarrow a A_{j 1} \cdots A_{j m} \text { in } P, \\
& h\left(\left[A_{i}, a\right]\right)=\tilde{a}_{1} \tilde{a}_{2}{ }^{i} \tilde{a}_{1} \quad \text { if } A_{i} \rightarrow a \text { in } P \text { and } i \neq 1, \\
& h\left(\left[A_{1}, a\right]\right)=\varnothing \quad \text { if } A_{1} \rightarrow a \text { in } P .
\end{aligned}
$$

It suffices to show that $L\left(G_{0}\right)=h^{-1}\left(\phi D_{2}\right)$ holds.

We claim the following: for $b_{1}, \cdots, b_{k}$ in $T^{\prime}, A_{i 1}, \cdots, A_{i r}$ in $N-\left\{A_{1}\right\}$, we have

$$
A_{1} \Longrightarrow{ }_{L}^{k} b_{1} \cdots b_{k} A_{i 1} \cdots A_{i r}(r \geq 0) \text { in } G_{0} \text { iff }
$$

and

(1) $h\left(b_{1} \cdots b_{k}\right)=\phi y_{1} \cdots y_{k}$ is a prefix of a word in $\phi D_{2}$,

(2) $\operatorname{red}\left(\phi y_{1} \cdots y_{k}\right)=\varnothing a_{1} a_{2}{ }^{i r} a_{1} \cdots a_{1} a_{2}{ }^{i 1} a_{1}$, where "red" is a mapping defined by

$$
\begin{aligned}
& \operatorname{red}(e)=e, \\
& \operatorname{red}(\phi)=\phi,
\end{aligned}
$$

for $i=1,2$

$$
\begin{aligned}
& \operatorname{red}\left(x a_{i}\right)=\operatorname{red}(x) a_{i}, \\
& \operatorname{red}\left(x \tilde{a}_{i}\right)=\operatorname{red}(x) \tilde{a}_{i} \quad \text { if } \operatorname{red}(x) \text { not in }\left\{a_{1}, a_{2}, \tilde{a}_{1}, \tilde{a}_{2}\right\} *\left\{a_{i}\right\}, \\
& \operatorname{red}\left(x \tilde{a}_{i}\right)=x^{\prime} \text { if } \operatorname{red}(x)=x^{\prime} a_{i} .
\end{aligned}
$$

(Note that $\Rightarrow_{L}^{k}$ indicates the $k$ step left-most derivation, i.e., $k$ consecutive rewriting steps in which the left-most nonterminal is always rewritten, and it is well-known that any word generated by a simple deterministic grammar has the unique left-most derivation for it. Further, from the property of a simple deterministic grammar, the length of a word generated exactly equals to the number of derivation steps used. A mapping image $\operatorname{red}(w)$, the reduced word, is the final resultant obtained by repeatedly cancelling all pairs $a_{i} \tilde{a}_{i}$.)

It should be noted that the claim suffices to prove the lemma. We shall prove the claim by induction on the length of dervation steps.

[k=1] Suppose that $A_{1} \Rightarrow b_{1}$ or $A_{1} \Rightarrow b_{1} A_{i 1} \cdots A_{i r}$. There exists $A_{1} \rightarrow b_{1}$ or $A_{1} \rightarrow b_{1} A_{i 1} \cdots$ $A_{i r}$ in $P^{\prime}$. Then, $h\left(b_{1}\right)=\phi$ or $h\left(b_{1}\right)=\phi a_{1} a_{2}{ }^{i r} a_{1} \cdots a_{1} a_{2}{ }^{i 1} a_{1}$. Clearly condition (2) holds for 
either case. Conversely assuming (1) and (2) for $k=1$ gives us that $h\left(b_{1}\right)=\phi y_{1}$ is a prefix of a word in $\phi D_{2}$ and $\operatorname{red}\left(\phi y_{1}\right)=\not a_{1} a_{2}{ }^{i r} a_{1} \cdots a_{1} a_{2}{ }^{i 1} a_{1}$. From the way of constructing $h$, if $\operatorname{red}\left(\phi y_{1}\right)=\phi(r=0)$, i.e., $y_{1}$ is in $D_{2}$, then we have $A_{1}->b_{1}$ is in $P^{\prime}$, leading to $A_{1} \Rightarrow b_{1}$. Otherwise, $h\left(b_{1}\right)=\phi y_{1}=\varnothing a_{1} a_{2}{ }^{i r} a_{1} \cdots a_{1} a_{2}{ }^{i 1} a_{1}$ implies that $A_{1} \rightarrow b_{1} A_{i 1} \cdots A_{i r}$ is in $P^{\prime}$. This verifies the case $k=1$.

[Induction step] Suppose that $A_{1} \Rightarrow_{L}^{k} b_{1} \cdots b_{k} A_{i 1} \cdots A_{i r}(r \geq 1)$ and $A_{i 1} \rightarrow b_{k+1} A_{j 1} \cdots A_{j m}(m \geq 0)$ is used at the $(k+1)$-th step. Let $h\left(b_{k+1}\right)=y_{k+1}$. By the induction hypothesis,

$$
\begin{aligned}
& \operatorname{red}\left(\phi y_{1} \cdots y_{k}\right)=\not a_{1} a_{2}{ }^{i r} a_{1} \cdots a_{1} a_{2}{ }^{i 1} a_{1} . \text { Then, we have } \\
& \begin{aligned}
\operatorname{red}\left(h\left(b_{1} \cdots b_{k+1}\right)\right) & =\operatorname{red}\left(\phi y_{1} \cdots y_{k+1}\right) \\
& =\varnothing a_{1} a_{2}{ }^{i r} a_{1} \cdots a_{1} a_{2}{ }^{i 2} a_{1} a_{1} a_{2}{ }^{j m} a_{1} \cdots a_{1} a_{2}{ }^{j 1} a_{1} .
\end{aligned}
\end{aligned}
$$

(Note that $y_{k+1}=\tilde{a}_{1} \tilde{a}_{2}{ }^{i 1} \tilde{a}_{1} a_{1} a_{2}{ }^{j m} a_{1} \cdots a_{1} a_{2}{ }^{j 1} a_{1}$.)

This also implies that $h\left(b_{1} \cdots b_{k+1}\right)$ is a prefix of a word in $\phi D_{2}$. Since $A_{1} \Rightarrow_{L}^{k+1} b_{1} \cdots$ $b_{k+1} A_{j 1} \cdots A_{j m} A_{i 2} \cdots A_{i r}$, the 'only if' part of the proof is proved.

Conversely, suppose that we have $h\left(b_{1} \cdots b_{k+1}\right)=\phi y_{1} \cdots y_{k+1}$ is a prefix of a word in $\phi D_{2}$ and $\operatorname{red}\left(\phi y_{1} \cdots y_{k+1}\right)=\varnothing a_{1} a_{2}{ }^{i r} a_{1} \cdots a_{1} a_{2}{ }^{i 1} a_{1}$. From the construction of $h$, we have a partition:

$$
\begin{aligned}
& \operatorname{red}\left(\phi y_{1} \cdots y_{k}\right)=\phi a_{1} a_{2}{ }^{i r} a_{1} \cdots a_{1} a_{2}{ }^{i p} a_{1}, \\
& \operatorname{red}\left(y_{k+1}\right)=h\left(b_{k+1}\right)=\tilde{a}_{1} \tilde{a}_{2}{ }^{t} \tilde{a}_{1} a_{1} a_{2}{ }^{i s} a_{1} \cdots a_{1} a_{2}{ }^{i 1} a_{1},
\end{aligned}
$$

where there exists $A_{t} \rightarrow b_{k+1} A_{i 1} \cdots A_{i s}$ in $P^{\prime}$.

But, since $\operatorname{red}\left(\phi y_{1} \cdots y_{k+1}\right)$ is a word of the form $\phi a_{1} a_{2}{ }^{i r} a_{1} \cdots a_{1} a_{2}{ }^{i 1} a_{1}$ there must be some cancellation between the two, which implies that $i p=t$. By the induction hypothesis,

$$
A_{1} \Longrightarrow{ }_{L}^{k} b_{1} \cdots b_{k} A_{t} \cdots A_{i r},
$$

and applying $A_{t} \rightarrow b_{k+1} A_{i 1} \cdots A_{i s}$, we have

$$
A_{1} \Longrightarrow{ }_{L}^{k+1} b_{1} \cdots b_{k+1} A_{i 1} \cdots A_{i s} \cdots A_{i r} .
$$

This completes the proof.

Communicated by T. Kitagawa

Received September 9, 1985 